\title{
Aspectos farmacológicos da terapia medicamentosa utilizada para a leishmaniose cutânea: uma revisão de literatura
}

\author{
Pharmacological aspects of drug therapy used for cutaneous leishmaniasis: a literature \\ review
}
Aspectos farmacológicos de la farmacoterapia utilizada para la leishmaniasis cutánea: una revisión de la literatura

Claudia Dantas Comandolli-Wyrepkowski ${ }^{1 *}$, Andréia da Costa Paz ${ }^{2}$, Bruno Bezerra Jensen ${ }^{1}$, Antonia Maria Ramos Franco'.

\section{RESUMO}

Objetivo: Descrever o arsenal terapêutico utilizado para o tratamento da Leishmaniose Cutânea (LC), revisando a literatura científica que tenha abordagem nos aspetos farmacológicos. Métodos: Revisão publicados em revistas indexadas em bases de dados como ScienceDirect, PubMed e Scielo, e manuais publicados pelo Ministério da Saúde e OMS, sendo os critérios: ser publicados entre os últimos 20 anos, que abordassem estudos com a farmacoterapia atual e alternativas farmacológicas para o tratamento da LC. Resultados: Foram encontrados 1957 artigos, sendo 1690 artigos no ScienceDirect, 206 no PubMed e 61 no Scielo. Artigos que relatavam novos fármacos promissores para LC, ou associações de fármacos já utilizados na terapia convencional com novas substâncias correspondiam a $27,59 \%$ do total. Outros aspectos da farmacoterapia amplamente abordados foram inovações no esquema terapêutico ou formulação, mas poucos trabalhos relatavam o mecanismo de ação. Fármacos como antimoniato de meglumina, isotionato de pentamidina e anfotericina $B$ são os mais utilizados na terapia da LC, e possíveis mecanismos de ação na célula de Leishmania foram revisados. Considerações finais: $O$ arsenal terapêutico para a LC ainda é limitado, e pouca inovação tem ocorrido nos últimos 20 anos, embora substâncias de origem natural vêm despertando interesse como possíveis novos alvos terapêuticos.

Palavras-chave: Leishmaniose, Antiparasitários, Farmacologia, Efeito dos fármacos.

\begin{abstract}
Objective: To describe the therapeutic arsenal used for the treatment of Cutaneous Leishmaniasis (LC), reviewing the scientific literature that addresses pharmacological aspects. Methods: Review published in journals indexed in databases such as ScienceDirect, PubMed and Scielo, and manuals published by the Ministry of Health and WHO, with the following criteria: to be published within the last 20 years, covering studies with current pharmacotherapy and pharmacological alternatives for the treatment of LC. Results: 1957 articles were found, 1690 articles in ScienceDirect, 206 in PubMed and 61 in Scielo. Articles that reported promising new drugs for LC, or combinations of drugs already used in conventional therapy with new substances corresponded to $27.59 \%$ of the total. Other aspects of pharmacotherapy widely addressed were innovations in the therapeutic scheme or formulation, but few studies reported the mechanism of action. Drugs such as meglumine antimoniate, pentamidine isothionate and amphotericin B are the most used in LC therapy, and possible mechanisms of action in the Leishmania cell have been reviewed. Final considerations: The therapeutic arsenal for LC is still limited, and little innovation has occurred in the last 20 years, although substances of natural origin have been attracting interest as possible new therapeutic targets.
\end{abstract}

Keywords: Leishmaniasis, Antiparasitic drugs, Pharmacology, Drug effects.

1 Instituto Nacional de Pesquisas da Amazônia, Manaus - AM. * E-mail: klaudiadcw@gmail.com

2 Universidade do Estado do Amazonas, Manaus - AM. 


\section{RESUMEN}

Objetivo: describir el arsenal terapéutico utilizado para el tratamiento de la leishmaniasis cutánea (LC), revisando la literatura científica que aborda los aspectos farmacológicos. Métodos: Esta es una revisión de artículos científicos publicados en revistas indexadas en bases de datos como ScienceDirect, PubMed y Scielo, y manuales publicados por el Ministerio de Salud y la OMS, con los siguientes criterios: se publicará entre los últimos 20 años, abordando estudios con farmacoterapia actual y alternativas farmacológicas para el tratamiento de LC. Resultados: se encontraron 1957 artículos, 1690 artículos en ScienceDirect, 206 en PubMed y 61 en Scielo. Los artículos que informaron nuevos medicamentos prometedores para LC o combinaciones de medicamentos ya utilizados en terapia convencional con nuevas sustancias correspondieron al $27.59 \%$ del total. Otros aspectos de la farmacoterapia ampliamente abordados fueron las innovaciones en el esquema o formulación terapéutica, pero pocos estudios informaron el mecanismo de acción. Los fármacos como el antimonio de meglumina, el isotionato de pentamidina y la anfotericina $B$ son los más utilizados en la terapia de LC, y se han revisado los posibles mecanismos de acción en la célula de Leishmania. Consideraciones finales: El arsenal terapéutico para la LC todavía es limitado, y se ha producido poca innovación en los últimos 20 años, aunque las sustancias de origen natural han estado atrayendo el interés como posibles nuevos objetivos terapéuticos.

Palabras clave: Leishmaniasis, Fármacos antiparasitarios, Farmacología, Efectos de los fármacos.

\section{INTRODUÇÃO}

Leishmanioses são doenças infecto-parasitárias, não contagiosas, sendo causadas por protozoários de diferentes espécies do gênero Leishmania, e que possuem a capacidade de invadir e reproduzir-se dentro das células que fazem parte do sistema imunológico do hospedeiro infectado (PACE D, 2014).

Os parasitos possuem ciclo de vida heteroxênico, alternando estágios de vida entre hospedeiros mamíferos e invertebrados, sendo transmitidos ao homem durante o repasto sanguíneo do vetor invertebrado (GONTIJO B e CARVALHO MLR, 2003)

A Leishmaniose Tegumentar (LT) constitui um problema de saúde pública em 98 países (ALVAR J, et al., 2012), distribuídos em quatro continentes (Américas, Europa, África e Ásia), com registro anual de dois milhões de novos casos registrados por ano e mais de 15 milhões de pessoas infectadas no Mundo. Em 2018 foram notificados mais de 46 mil novos casos da leishmaniose cutânea e mucosa nas Américas, sendo no Brasil a maior concentração de casos, com cerca de 35\% (PAHO, 2019).

No espectro clínico da Leishmaniose Tegumentar Americana, os pacientes podem apresentar a forma cutânea, que é caracterizada por lesões localizadas na pele, que pode curar-se espontaneamente ou evoluir para lesões crônicas, com cicatrizes desfigurantes. As lesões cutâneas constituem úlceras rasas, circulares com bordas elevadas e bem definidas e com o assoalho da úlcera de aspecto granular (SILVEIRA FT, et al., 2004).

A evolução da lesão da Leishmaniose Cutânea, no homem, apresenta variantes. Frequentemente a hiperplasia histiocitária e a inflamação local causam a necrose (morte celular) da epiderme e das camadas subjacentes, na área parasitada, em vista das perturbações mecânicas e tróficas decorrentes desse crescimento patológico (MARTINS ALGP, et al., 2014).

Os tratamentos de primeira escolha para muitas formas de LTA são injeções diárias de antimoniais pentavalentes, como o antimoniato de N-metilgucamina (Glucantime ${ }^{\circledR}$ ) e o estibogluconoato de Sódio (Pentostan ${ }^{8}$ ) (TIUMAN TS, et al., 2011). Drogas como isotionato de pentamidina, miltefosina, anfotericina B e anfotericina $B$ lipossomal, constituem opções terapêuticas aos casos de baixa eficiência dos tratamentos (CARVALHO SH, et al., 2019).

Para avaliação do critério de cura, utiliza-se o exame clínico. Recomenda-se acompanhamento mensal por três meses consecutivos e, após a cura clínica, seguimento até 12 meses após o término do tratamento 
(GONTIJO B e CARVALHO MLR, 2003). Na forma cutânea o critério de cura é definido pelo aspecto clínico das lesões: reepitelização das lesões ulceradas ou não-ulceradas, regressão total da infiltração e eritema, até três meses após a conclusão do esquema terapêutico.

Os fármacos conhecidos e utilizados para o tratamento de leishmanioses tegumentares apresentam uma série de problemas, que incluem resistência do parasita e indução de efeitos colaterais, que limitam a utilização e, principalmente, a eficácia. Além disso, todos os fármacos que estão disponíveis atualmente são de administração parenteral, o que exige colaboração do paciente e, que infelizmente, muitos abandonam o tratamento, fator que favorece o aparecimento de cepas resistentes (SILVA-LOPEZ RE, 2010).

As dificuldades quanto à administração e a duração do tratamento com os fármacos mais utilizados para as leishmanioses, como os antimoniais pentavalentes, paralelamente aos seus efeitos colaterais, têm estimulado pesquisadores a buscar novas substâncias com ação contra Leishmania, assim como também formas farmacêuticas que possuam maior facilidade de administração e menores efeitos colaterais.

Neste contexto, as estratégias com base na melhoria de medicamentos existentes são mais bemsucedidas do que aquelas com base no design de novas entidades químicas. Avanços incluem 0 desenvolvimento de formulações mais eficazes e mais seguras para drogas leishmanicida existentes, o uso de drogas originalmente concebido e avaliadas para doenças não relacionadas, novas combinações de fármacos e protocolos terapêuticos (SINGH N, et al., 2012).

Contudo, a problemática no desenvolvimento de novas drogas eficientes para o tratamento da Leishmaniose inclui ainda o fato de que as diferentes formas de manifestação da doença exigem diferentes mecanismos farmacocinéticos das drogas utilizadas (BERMAN J, et al., 2005); a grande variabilidade da sensibilidade das drogas nas diferentes espécies de Leishmania conhecidas que infectam o homem; diferenças metabólicas entre promastigotas e amastigotas, havendo assim a necessidade de estudos mais aprofundados e associações entre as diversas áreas do conhecimento para o desenvolvimento e/ou melhoramento dos fármacos.

Assim, sendo a Leishmaniose uma doença negligenciada de grande importância nacional e até mundial, faz-se necessário compreender melhor os aspectos do tratamento e a farmacoterapia preconizada e utilizada atualmente, assim como as novas descobertas de substâncias bioativas que podem ser candidatas a novos fármacos. O presente trabalho teve por objetivo principal identificar na literatura o arsenal farmacoterapêutico utilizando atualmente para o tratamento da Leishmaniose Cutânea, analisando e organizando os aspectos farmacológicos das drogas utilizadas e preconizadas pelos orgãos de saúde.

\section{MÉTODOS}

O trabalho trata-se de um estudo do tipo revisão de literatura integrativa, norteada para responder à pergunta "Quais aspectos farmacológicos das terapias atualmente utilizadas para a Leishmaniose Cutânea?". Foi realizado uma revisão com base em artigos científicos publicados em periódicos indexados, selecionados através de buscas no banco de dados: Scientific Eletronic Library Online (SciELO), Publisher Medline (PubMed) e ScienceDirect.

Para a pesquisa foram utilizadas a associação das palavras-chave: "Cutaneous Leishmaniasis", "treatment", "therapy", "drugs" inseridos nos campos do título, resumo, palavras-chave ou texto, e associados com inserção de "Leishmaniasis" ou "Leishmania" no título da busca (Figura 1). A pesquisa utilizou filtro de tempo de 20 anos (selecionando artigos entre o ano 2000 a 2020).

Os critérios de inclusão foram ser artigos publicados em português, inglês ou espanhol, sem restrição à determinada região ou país, que abordassem aspectos farmacológicos do tratamento utilizado para a Leishmaniose Cutânea, podendo ser incluídos no termo farmacológico: ensaios pré-clínicos e clínicos, desenvolvimento e avaliação de formulações farmacêuticas e vias de administração, parâmetros farmacocinéticos e farmacodinâmicos, mecanismo de ação e efeitos adversos. Foram excluídos artigos que abordassem o tratamento de formas clínicas relacionadas a Leishmaniose Visceral e tratamentos que não fossem de natureza medicamentosa. 
aspectos farmacológicos do tratamento com este fármaco. Outros fármacos citados na literatura que vêm sendo utilizados para tratamento da LC foram a miltefosina $(15,43 \%)$ paromomicina $(8,64 \%)$, alopurinol $(1,68 \%)$ e azitromicina (1,27\%) (TEIXEIRA AC, et al., 2007; WIJNANT GJ, et al., 2018).

Estudos de revisão e experimentais que continham outros fármacos promissores, ou associações de diferentes fármacos já preconizados e utilizados com novas substâncias correspondiam a 27,59\% dos artigos encontrados nas bases de dados da pesquisa.

Substâncias bioativas vêm sendo alvo de estudos pré-clínicos como candidatas a novos fármacos para o tratamento da LC, e a literatura relata avaliações também sobre a eficácia com o uso concomitante de um fármaco preconizado para o tratamento associado a um tratamento alternativo (como com substâncias de origem natural), utilizando novas formulações ou vias de administração (ARAUJO IAC, et al., 2019).

Os primeiros medicamentos utilizados para LC baseavam-se em sais de antimônio trivalentes (Sb III), usando trivalentes, mais especificamente antimônio tartarato de potássio ou tártaro emético, mas a administração destes compostos foi descontinuada após o desenvolvimento de drogas menos tóxicas à base de antimoniais pentavalente (CARVALHO SH, et al., 2019).

O ácido estibônico complexado a carboidratos (duas moléculas de ácido glicônico) é utilizado para o tratamento das leishmanioses em países de língua inglesa (RATH S, 2003). Em países de língua francesa, espanhola e no Brasil, o fármaco utilizado é o antimoniato de meglumina, também um antimonial pentavalente (Glucantime®) (BERMAN J, 2005).

No Brasil, a única formulação disponível é o antimoniato de N-metil glucamina, que vem sendo distribuída pelo Ministério da Saúde (BRASIL, 2017). A Organização Mundial de Saúde (2012), recomenda o tratamento de LTA com antimonial pentavalente por via endovenosa ou intramuscular, com a dose recomendada de 20 $\mathrm{mg} / \mathrm{kg}$ por dia, com tratamento durante 28 dias.

Apesar dos mais de 60 anos de uso e da eficácia comprovada dos antimoniais pentavalentes, pouco se sabe sobre o seu mecanismo de ação. FRÉZARD F, et al. (2009) sugerem que isso ocorre principalmente devido ao estado amorfo destes compostos.

Contudo, há uma concordância de que esta droga interfere na bioenergética das formas amastigotas de Leishmania, atuando na glicólise e na oxidação dos ácidos graxos (YAN SC, et al., 2003). Os trabalhos que discutem o mecanismo de ação dos antimoniais são escassos quando comparado com outros parâmetros farmacológicos, e mecanismos propostos até o presente momento estão apresentados no Quadro 1, indicando que os antimoniais podem atuar sobre diversas vias do metabolismo do parasita.

Os compostos antimoniais, por serem pouco absorvidos no epitélio gastrointestinal e irritantes para esse sistema, são administrados parenteralmente. Estudos indicam que os antimoniais trivalentes inibem a fosfofrutoquinase, inibindo dessa forma a conversão de frutose-6-fosfato a frutose 1,6-difosfato nas vias glicolíticas.

Sua ação ocorre tanto nas células do parasita, quanto nas células dos mamíferos, porém a enzima fosfofrutoquinase dos mamíferos é cerca de 80 vezes menos sensível que a do parasita, permitindo que 0 antimonial exerça seus efeitos e modo seletivo (CATALDO Jl, et al., 2018).

Em geral, os antimoniais pentavalentes são bem tolerados, mas alguns efeitos colaterais podem ocorrer como dor no local da injeção, disfunção gastrintestinal, dores musculares difusas, enrijecimento das articulações, arritmias e pancreatite (FERREIRA CC, et al., 2012). A elevação das transaminases hepáticas também foi observada, mas de maneira reversível, cessando com o fim do tratamento (ARBOLEDA M, et al., 2019). 
Quadro 1 - Aspectos da farmacodinâmica dos principais fármacos preconizados e utilizados para a terapia da Leishmaniose Cutânea (LC).

\begin{tabular}{|c|c|c|c|}
\hline Fármaco & Mecanismo de ação & $\begin{array}{l}\text { Atividade na célula de } \\
\text { Leishmania }\end{array}$ & Referência \\
\hline \multirow{4}{*}{$\begin{array}{c}\text { Antimoniais } \\
\text { entavalentes } \\
\text { (estibogluconato de } \\
\text { sódio Pentostan } \AA \text { ] e } \\
\text { antimoniato de N-metil- } \\
\text { glucamina } \\
\text { [Glucantime } \AA \text { ], }\end{array}$} & $\begin{array}{l}\text { inibição da glicólise e na } \beta \text { - } \\
\text { oxidação de ácidos graxos. }\end{array}$ & depleção de ATP & $\begin{array}{l}\text { BERMAN J, } \\
2005\end{array}$ \\
\hline & $\begin{array}{c}\text { interferência na síntese de tióis } \\
\text { (Glutationa - GSH, Cisteína - } \\
\text { Cys, Cisteinil-glicina - Cys-Gly e } \\
\left.\text { Tripanotiona - } \mathrm{T}(\mathrm{SH})_{2}\right)\end{array}$ & indução da apoptose & $\begin{array}{l}\text { YAN SC et } \\
\text { al., } 2003\end{array}$ \\
\hline & $\begin{array}{c}\text { indução do efluxo de tióis } \\
\text { intracelulares }\end{array}$ & $\begin{array}{l}\text { inibição da tripanotiona } \\
\text { redutase }\end{array}$ & $\begin{array}{l}\text { CATALDO Jl, } \\
\text { et al., } 2018\end{array}$ \\
\hline & $\begin{array}{l}\text { Ação similar a endonucleases, } \\
\text { fragmentando o DNA }\end{array}$ & indução da apoptose & $\begin{array}{l}\text { RATH S, et } \\
\text { al., } 2003\end{array}$ \\
\hline \multirow{4}{*}{$\begin{array}{l}\text { Pentamidina } \\
\text { (isotionato de } \\
\text { pentamidina) }\end{array}$} & $\begin{array}{l}\text { perturbação mitocondrial } \\
\text { via moléculas mediadoras }\end{array}$ & $\begin{array}{l}\text { inibição do metabolismo } \\
\text { mitocondrial e inibidor } \\
\text { competitivo de arginina }\end{array}$ & $\begin{array}{l}\text { BASSELIN } \\
\text { M, et al. } 2002\end{array}$ \\
\hline & $\begin{array}{l}\text { interferem na síntese de } \\
\text { poliaminas }\end{array}$ & $\begin{array}{l}\text { inibem a utilização de S- } \\
\text { adenosil-L metionina, } \\
\text { impedindo a síntese de } \\
\text { moléculas importantes } \\
\text { para a manutenção da } \\
\text { vida do parasita }\end{array}$ & $\begin{array}{l}\text { SILVA- } \\
\text { LOPES RE, } \\
2010\end{array}$ \\
\hline & $\begin{array}{l}\text { compete com poliaminas pelo } \\
\text { ácido nucleico de ligação e pode } \\
\text { se ligar ao DNA cinetoplástico }\end{array}$ & $\begin{array}{l}\text { interferem na replicação e } \\
\text { transcrição em nível } \\
\text { mitocondrial }\end{array}$ & $\begin{array}{l}\text { COELHO } \\
\text { AC, et } \\
\text { al.,2003 }\end{array}$ \\
\hline & $\begin{array}{l}\text { aumentam a eficácia dos } \\
\text { inibidores do Complexo II da } \\
\text { cadeia respiratória mitocondrial }\end{array}$ & $\begin{array}{c}\text { diminuição do potencial } \\
\text { de membrana } \\
\text { mitocondrial }\end{array}$ & $\begin{array}{l}\text { SINGH N, et } \\
\text { al.,2012 }\end{array}$ \\
\hline \multirow{2}{*}{ Anfotericina B } & $\begin{array}{l}\text { ligação ao ergosterol - via } \\
\text { biossintética de esteróis }\end{array}$ & $\begin{array}{c}\text { alteração de } \\
\text { permeabilidade de } \\
\text { membrana e do equilíbrio } \\
\text { osmótico do parasita }\end{array}$ & $\begin{array}{l}\text { TIUMAN TS, } \\
\text { et al., } 2011\end{array}$ \\
\hline & $\begin{array}{l}\text { despolarização da membrana e } \\
\text { um aumento na permeabilidade } \\
\text { da membrana a prótons e } \\
\text { cátions monovalentes }\end{array}$ & $\begin{array}{l}\text { alteram o equilíbrio de } \\
\text { íons e conduzem à morte } \\
\text { celular }\end{array}$ & $\begin{array}{l}\text { WIJNANT } \\
\text { GJ, et al., } \\
2018\end{array}$ \\
\hline
\end{tabular}

Fonte: Comandolli-Wyrepkowski CD, et al., 2020.

O Glucantime ${ }^{\circledR}$ (antimoniato de meglumina) é fornecido em ampolas de $5 \mathrm{~mL}$ contendo $300 \mathrm{mg} / \mathrm{mL}$ de antimoniais de meglumina (AM), teoricamente equivalente a uma concentração de princípio ativo $\mathrm{Sb}^{5}$ de 81 $\mathrm{mg} / \mathrm{mL}$ e uma dose total de $\mathrm{Sb}^{5}$ de $405 \mathrm{mg}$ por ampola.

A dose prescrita deve ser com base na quantidade de antimônio pentavalente presente, mas discrepâncias entre as concentrações totais de $\mathrm{AM}$ e $\mathrm{Sb}^{5}$ nas ampolas podem levar a erros de prescrição (CARVALHO SH, et al., 2019). O padrão regime terapêutico para adultos e crianças varia de 10 a $20 \mathrm{mg} \mathrm{Sb} / \mathrm{kg} / \mathrm{dia}$ administrado por 20 a 30 dias consecutivos por meio de via intravenosa (IV) ou intramuscular (como primário), com tratamento repetido se necessário (BRASIL, 2017). 
A Anfotericina B, antibiótico macrolídeo poliênico de reconhecida ação leishmanicida, é a droga de segunda escolha, empregada quando não se obtém resposta ao tratamento com antimonial ou na impossibilidade de seu uso (CHAVEZ-FUMAGALLI MA, et al., 2015). Foi desenvolvida formulações lipossomais de Anfotericina $B$ que têm diminuído seus efeitos colaterais, e é recomendada

A anfotericina é considerada a droga de segunda escolha quando não se obtém resposta ao tratamento com o antimonial pentavalente ou na impossibilidade de seu uso. É contra-indicada a administração da anfotericina B em cardiopatas, hepatopatas e, especialmente, nefropatas (NEVES LO, et al., 2011). Os efeitos adversos mais frequentes são febre, náuseas, vômitos, hipopotassemia e flebite no local da infusão. Uma formulação da anfotericina $B$ à base de lipossomas (AmBisome®), depois de testes clínicos bem sucedidos, chegou a ser aprovada pela FDA (Food and Drug Administration) para o tratamento do calazar (WIJNANT GJ, et al., 2018).

O tratamento com anfotericina $B$ recomendado para Leishmaniose Cutânea é por via intravenosa, sendo recomendada a infusão lenta, com velocidade máxima de $2,5 \mathrm{mg} / \mathrm{kg} / \mathrm{h}$. A dose recomendada é $3 \mathrm{a} 5 \mathrm{mg} / \mathrm{kg} / \mathrm{dia}$, geralmente sendo 7 doses recomendadas, mas podendo varias de 2 a 10 doses (WORTMANN G, et al., 2010). Sua ação baseia-se na interação com o ergosterol da membrana celular, formando poros que alteram a permeabilidade celular e o balanço iônico, causando a morte da célula (TIUMAN TS, et al., 2011). Esses defeitos causam despolarização da membrana e um aumento na permeabilidade da membrana a prótons e cátions monovalentes. As interações intermoleculares de ligação de hidrogênio entre os grupos hidroxila, carboxila e amino estabilizam o canal em sua forma aberta, destruindo a atividade e permitindo que 0 conteúdo citoplasmático vaze, levando à morte celular (WIJNANT GJ, et al., 2018).

As pentamidinas são diamidinas aromáticas que são utilizadas como drogas de segunda escolha no tratamento da LC em áreas endêmicas dos continentes americano, asiático e africano (LIMA EB, et al., 2007). A descoberta da atividade antiprotozoária das diamidinas foi consequente a procura por compostos hipoglicemiantes que pudessem comprometer o metabolismo energético de parasitas (NEVES LO, et al., 2011). O mecanismo de ação da pentamidina ocorre por inibição de diferentes processos celulares, ainda não são totalmente elucidados. O isotionato de pentamidina é administrado por via intramuscular ou, de preferência, por infusão intravenosa, na dose de 2-4 mg/kg/dia, em dias alternados, sendo duas a quatro aplicações, embora há relatos de tratamentos com uma única dose de $7 \mathrm{mg} / \mathrm{kg}$ (OLIVEIRA LF, et al., 2011). Porém, efeitos adversos graves, como diabetes mellitus, hipoglicemia grave, choque, miocardite e toxicidade renal, limitam seu uso (NEVES LO, et al., 2011).

Os trabalhos de Basselin $\mathrm{M}$ et al. (2002) mostraram que a além da pentamidina atuar na inibição do metabolismo mitocondrial em espécies de Leishmania, ela também atua como um inibidor competitivo de arginina, capturando promastigotas e amastigotas, supondo que a droga entra tanto em promastigotas quanto em amastigotas via células mediadoras, cujo processo reconhece diamidinas com alta afinidade, sugerindo que a energia metabólica é necessária para o transporte de poliaminas em ambas as formas do parasita. $O$ mesmo foi observado posteriormente por Silva-López RE (2010), onde demonstrou que as diamidinas que constituem a droga interferem na síntese de poliaminas, inibindo a utilização de S-adenosil-L metionina por inibir enzimas como a ornitina descarboxilase e a espermidina sintetase, impedindo, assim, a síntese de moléculas importantes para a manutenção da vida do parasita.

Os estudos de Coelho AC, et al. (2003) indicaram ainda que a pentamidina compete com poliaminas pelo ácido nucleico de ligação e pode também se ligar preferencialmente ao DNA cinetoplástico interferindo na replicação e transcrição em nível mitocondrial em Leishmania major. Os resultados do trabalho de Mukherjee A, et al. (2006) sugeriram que a energia é necessária para a entrada da droga no promastigota, e que a atividade metabolizante da mitocôndria desempenha um papel importante na acumulação de pentamidina. Em seu trabalho de revisão sobre os atuais tratamentos da leishmaniose Singh N, et al. (2012) ressaltam que a pentamidina acumula-se na mitocôndria e aumenta a eficácia dos inibidores do Complexo II da cadeia respiratória mitocondrial, sugerindo atividade leishmanicida devido a diminuição do potencial de membrana mitocondrial.

A Azitromicina é o primeiro antibiótico da subclasse dos macrolídeos, conhecida como azalídeos, um conhecido antibiótico, foi avaliada clinicamente em 20 pacientes com Leishmaniose Cutânea, mostrando uma 
taxa de cura de $85 \%$ e testes em hamster também indicaram que a azitromicina foi ativa contra L. (V.) braziliensis (SINAGRA A, 2007). Entretanto, um estudo clínico realizado em Manaus, demonstrou baixa eficiência no tratamento de pacientes infectados por L. (V.) guyanensis, a espécie de maior predominância na região (TEIXEIRA AC, et al., 2007).

A azitromicina também já se mostrou ineficaz em pacientes com LC infectados com L. braziliensis, de um Multicêntrico de Minas Gerais (TOLEDO-JUNIOR A, et al., 2014). Logo, devido ao seu desempenho terapêutico sugeriu-se o uso da azitromicina associado a outro medicamento para avaliar o seu perfil clínico.

A Paromomicina é um antibiótico da classe aminoglicosídeo e é um dos medicamentos com atividade terapêutica de segunda linha para as Leishmanioses Cutânea e Visceral, principalmente pela segurança, baixo custo e curto intervalo de aplicação. Esse fármaco atua no bloqueio da síntese de proteínas por meio da ligação ao 16S RNA ribossômico (KHAN W, et al., 2013).

Heidari-Kharaji M, et al. (2016), comprovaram que no uso de uma Nanopartícula lipídica sólida com paromomicina testada em BALB/c infectados com Leishmania tropica, pode ser segura, viável e eficaz no controle da carga parasitária, formulação está contribuindo com a liberação controlada desse fármaco.

A paromomicina tópica também já se mostrou eficaz e segura em pacientes pediátricos com LC do Velho Mundo. Um dos estudos é de Chacko A, et al. (2016), onde tratou uma criança infectada com L. tropica com esse antibiótico, após a falha no tratamento com fluconazol, abrindo mais uma opção de tratamento menos invasivo.

A miltefosina, uma droga utilizada inicialmente para o tratamento de câncer, também têm demonstrado eficiência no tratamento das Leishmanioses, dependendo muitas vezes das espécies causadoras (SOTO J, et al., 2001). Este fármaco é o único de administração oral para essa doença, normalmente o seu uso é por combinação com outros medicamentos, como a Pentamidina intralesional que já apresentou uma taxa de cura de $92 \%$ para L. braziliensis na Bolívia (SOTO J, et al., 2018). Contudo, o seu mecanismo de ação não é bem compreendido (ALONSO L. et al., 2019).

Outras drogas vêm sendo recomendadas no tratamento da LTA, como o alopurinol. Utilizado para prevenir altos índices de ácido úrico, apresenta menos efeitos colaterais que as drogas comumente utilizadas (BERMAN J, 2005).

Com o objetivo de desenvolver drogas mais eficazes, de baixo custo e com reações adversas menos agressivas para o hospedeiro, novos alvos quimioterápicos para Leishmania têm sido investigados e novas abordagens para o desenvolvimento de fármacos devem ser consideradas.

Um dos importantes métodos de pesquisa para o desenvolvimento de novos medicamentos se baseia na busca de novos protótipos farmacêuticos encontrados principalmente em produtos de origem natural (ARAUJO IAC, et al., 2019). O estudo biomonitorado permite um isolamento mais rápido e eficaz do composto ativo, podendo-se ainda, introduzir modificações químicas nestes protótipos naturais com o objetivo de amplificar sua atividade específica ou mesmo reduzir seu efeito tóxico.

No Brasil, estudos com produtos naturais têm mostrado também o potencial de substâncias e óleos obtidos de espécies encontradas no país. Atividade leishmanicida foi detectada em benzofenonas preniladas isoladas de Garcinia brasiliensis (PEREIRA IO, et al., 2010) e também em óleo de copaíba extraído de diferentes espécies do gênero Copaifera (SANTOS AO, et al., 2008). Entre as plantas que estão despertando o interesse da continuidade de estudos, Kalanchoe pinnata tem se destacado por apresentar flavonoides com atividade anti-leishmania (ARAÚJO IAC, et al., 2019).

Apesar de encontrar na literatura uma diversidade de produtos naturais ou sintéticos que mostraram atividade inibitória contra Leishmania em sistemas in vitro, há pouca continuidade dos estudos para ensaios in vivo e clínicos para efetivamente transformar o conhecimento científico em tratamento alternativo.

A busca por novas substâncias para fins terapêuticos deve ser incentivada constantemente, pois deve ser levada em consideração a biodiversidade brasileira ser a maior do mundo, consecutivamente grandes oportunidades para explorar seus recursos para fins terapêuticos, principalmente para muitas doenças que carecem de medicamentos mais eficazes, como a leishmaniose cutânea. 


\section{CONSIDERAÇÕES FINAIS}

O tratamento da Leishmaniose Cutânea (LC) pode ser local ou de forma sistêmica, dependendo de como a lesão está evoluindo, de qual espécie de Leishmania está envolvida, e um grande volume de trabalhos experimentais buscam avaliar a eficácia de tratamentos com os fármacos convencionais em novas formulações farmacêuticas ou associados a outros compostos bioativos. Os principais fármacos recomendados para o tratamento da LC são os antimoniais, administrados vias intralesional e intramuscular, anfotericina B e a pentamidina, ambas também de uso parenteral. A busca por novos tratamentos que sejam menos tóxicos e facilmente biodisponíveis para tratar a população afetada pela Leishmaniose é imperativa e o conhecimento do mecanismo de ação pode auxiliar como guia para desenvolver novos fármacos.

\section{AGRADECIMENTOS E FINANCIAMENTO}

Agradecemos a pesquisadora Maricleide de Farias Naiff, do Laboratório de Leishmaniose e Doença de Chagas - INPA, e aos órgãos de fomento CAPES pela bolsa concedida à Claudia Dantas ComandolliWyrepkowski (Processo n. 88887.156153/2017-00) e FAPEAM pela bolsa concedida à Bruno Bezerra Jensen (Resolução n. 002/2006 POSGRAD 2017).

\section{REFERÊNCIAS}

1. ALONSO L, et al. Antileishmanial and cytotoxic activities of ionic surfactants compared to those of miltefosine. Colloids and Surfaces B: Biointerfaces, 2019;183: 110421.

2. ALVAR J. et al. Leishmaniasis Control Team. Leishmaniasis worldwide and global estimates of its incidence. PLoS One, 2012; 7(5).

3. ARAÚJO IAC, et al. Efficacy of lapachol on treatment of cutaneous and visceral leishmaniasis. Experimental Parasitology, 2019; 199:67-73.

4. ARBOLEDA M, et al. Successful treatment of cutaneous leishmaniasis with intralesional meglumine antimoniate: A case series. Journal of the Brazilian Society of Tropical Medicine. 2019; 52:e20180211.

5. BASSELIN M, et al. Resistance to Pentamidine in Leishmania mexicana Involves Exclusion of the Drug from the Mitochondrion. Antimicrobial agents and Chemotherapy, 2002; 46(2): 3731-3738.

6. BERMAN, J. Recent Developments in Leishmaniasis: Epidemiology, Diagnosis, and Treatment. Clin. Infect Dis., 2005; 7:33 - 38 .

7. BRASIL. Manual de Vigilância da Leishmaniose Tegumentar Americana. Ministério da Saúde, Secretaria de Vigilância em Saúde. Brasília: Editora do Ministério da Saúde, 2017.

8. CARVALHO SH, et al. American tegumentary leishmaniasis in Brazil: a critical review of the current therapeutic approach with systemic meglumine antimoniate and short-term possibilities for an alternative treatment. Tropical Medicine and International Health, 2019; 24(4): 380-391.

9. CATALDO JI, et al. Favorable responses to treatment with $5 \mathrm{mg} \mathrm{Sb} / \mathrm{kg} /$ day meglumine antimoniate in patients with American tegumentary leishmaniasis acquired in different Brazilian regions. Rev Soc Bras Med Trop, 2018; 51 (6):769780.

10. CHACKO A, et al. Case Report: Successful Treatment of Cutaneous Leishmaniasis with Topical Paromomicin in a Child after Treatment Failure with Systemic Fluconazole. American Journal of Tropical Medicine and Hygiene, 2016. 95(4): 793-794.

11. CHÁVEZ-FUMAGALLI MA, et al. New delivery systems for amphotericin $B$ applied to the improvement of leishmaniasis treatment. Revista da Sociedade Brasileira de Medicina Tropical, 201548 (3):235-242.

12. COELHO AC, et al. Functional genetic identification of PRP1, an ABC transporter superfamily member conferring pentamidine resistance in Leishmania major. Molecular \& Biochemical Parasitology, 2003; 130: 83-91.

13. FERREIRA CC, et al. Estudo sobre a Leishmaniose Tegumentar Americana com Enfoque na Farmacoterapia. Revista Científica do ITPAC, 2012, 5(4): 2-10.

14. FRÉZARD F, et al. Pentavalent Antimonials: New Perspectives for Old Drugs. Molecules, 2006; 14: $2317-2336$

15. GONTIJO B, CARVALHO MLR. Leishmaniose Tegumentar Americana. Revista da Sociedade Brasileira de Medicina Tropical, 2003; 36 (1): 71-8.

16. HEIDARI-KHARAJI M, et al. Solid lipid nanoparticle loaded with paromomycin: in vivo efficacy against Leishmania tropica infection in BALB/c mice model. Appl Microbiol Biotechnol; 2016; 100:7051-7060.

17. KHAN W et al. Bioanalytical method development, pharmacokinetics, and toxicity studies of paromomycin and paromomycin loaded in albumin microspheres. Drug Testing and Analysis; 2013; 5: 453-460.

18. LIMA EB, et al. Tratamento da Leishmaniose Tegumentar Americana. An. Bras. Dermatol., 2007; 82: 111-24.

19. MARTINS ALGP, et al. American tegumentary leishmaniasis: correlations among immunological, histopathological and clinical parameters. Anais Brasileiros de Dermatologia, 2014; 89 (1): 52-8.

20. MUKHERJEE A, et al. Roles for mitochondria in pentamidine susceptibility and resistance in Leishmania donovani. Molecular \& Biochemical Parasitology, 2006; 145:1-10. 
21. NEVES LO, et al. Estudo clínico randomizado comparando antimoniato de meglumina, pentamidina e anfotericina $B$ para o tratamento de leishmaniose cutânea ocasionada por Leishmania guyanensis. An Bras Dermatol, 2011; 86(6):1092-101.

22. OLIVEIRA LF, et al. Systematic review of the adverse effects of cutaneous leishmaniasis treatment in the New World. Acta Tropica; 2011; 118: 87-96.

23. ORGANIZAÇÃO MUNDIAL DE SAÚDE. 2012 In: Control of Leishmaniasis. Geneva: World Health Organization Disponível em: http://new. https://apps.who.int/iris/bitstream/handle/10665/44412/WHO_TRS_949 > Acesso em: 16 de jan 2020.

24. PACE. D. Leishmaniasis. Journal of Infection. V. 69, p. 10-18. 2014.

25. PAHO - PAN AMERICAN HEALTH ORGANIZATION - WORLD HEALTH ORGANIZATION. Leishmaniases: Epidemiological Report of the Americas. Report Leishmaniasis № 8, 2019.

26. PEREIRA, IO, et al. Leishmanicidal activity of benzophenones and extracts from Garcinia brasiliensis Mart. Fruits. Phytomedicine, 2010; 17:339-345.

27. RATH S, et al. Antimoniais empregados no tratamento da Leishmaniose: estado da Arte. Quimica Nova, 2003; 26(4):550-555.

28. SANTOS AO, et al. Effect of Brazilian copaiba oils on Leishmania amazonensis. Journal of Ethnopharmacology, 2008; 120 (2):204-208.

29. SILVA-LÓPEZ RE. Proteases de Leishmania: novos alvos para o desenvolvimento racional de fármacos. Química Nova, 2010; 33:1541 - 1543.

30. SILVEIRA FT, LAINSON R, CORBETT CEP. Clinical and Immunopathological Spectrum of American Cutaneous Leishmaniasis with Special Reference to the Disease in Amazonian Brazil - A Review. Memórias do Instituto Oswaldo Cruz, 2004; 99(3): 239-251.

31. SINAGRA A, et al. The activity of azithromycin against Leishmania (Viannia) braziliensis and Leishmania (Leishmania) amazonensis in the golden hamster model. Revista da Sociedade Brasileira de Medicina Tropical, $2007 ; 40$ (6): 627 630.

32. SINGH N, et al. Leishmaniasis: Current status of available drugs and new potential drug targets. Asian Pacific Journal of Tropical Medicine, 2012; 3: 485-497, 2012.

33. SOTO $\mathrm{J}$ et al. Treatment of American Cutaneous Leishmaniasis with Miltefosine, an Oral Agent. Clinical Infectious Diseases, 2001; 33: 57-61.

34. SOTO J, et al. Miltefosine Combined with Intralesional Pentamidine for Leishmania braziliensis Cutaneous Leishmaniasis in Bolivia. American Journal of Tropical Medicine and Hygiene; 2018; 99(5): 1153-1155.

35. TEIXEIRA AC, et al. Low efficacy of azithromycin to treat cutaneous leishmaniasis in Manaus, AM, Brazil. Rev. Inst. Med. trop., 2007; 49 (4): 235-238.

36. TIUMAN TS, et al. Recent advances in leishmaniasis treatment. International Journal of Infectious Diseases, 2011; 15: $525-32$.

37. TOLEDO-JUNIOR A. et al. Poor response to azithromycin in cutaneous leishmaniasis leading to a premature interruption of a multicentric phase III clinical trial in Brazil. Revista da Sociedade Brasileira de Medicina Tropical, 2014; 47(6):756-762.

38. WIJNANT GJ et al. Comparative efficacy, toxicity and biodistribution of the liposomal amphotericin B formulations

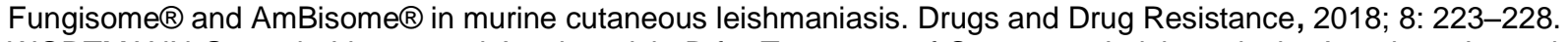

39. WORTMANN G, et al., Lipsosomal Amphotericin B for Treatment of Cutaneous Leishmaniasis. American Journal of Tropical Medicine and Hygiene, 2010; 83(5): 1028-1033.

40. YAN SC, et al. Reduction of pentavalent antimony by trypanothione and formation of a binary and ternary complex of antimony (III) and trypanothione. J. Biol. Inorg. Chem, 2003; 8:689-697. 